\title{
On the binomial edge ideal of a pair of graphs
}

\author{
Sara Saeedi Madani Dariush Kiani* \\ Department of Pure Mathematics \\ Faculty of Mathematics and Computer Science \\ Amirkabir University of Technology (Tehran Polytechnic) \\ 424, Hafez Ave., Tehran 15914, Iran \\ sarasaeedi@aut.ac.ir dkiani@aut.ac.ir
}

Submitted: Dec 16, 2012; Accepted: Feb 22, 2013; Published: Mar 1, 2013

Mathematics Subject Classifications: 13C05, 16E05, 05E40

\begin{abstract}
We characterize all pairs of graphs $\left(G_{1}, G_{2}\right)$, for which the binomial edge ideal $J_{G_{1}, G_{2}}$ has linear relations. We show that $J_{G_{1}, G_{2}}$ has a linear resolution if and only if $G_{1}$ and $G_{2}$ are complete and one of them is just an edge. We also compute some of the graded Betti numbers of the binomial edge ideal of a pair of graphs with respect to some graphical terms. In particular, we show that for every pair of graphs $\left(G_{1}, G_{2}\right)$ with girth (i.e. the length of a shortest cycle in the graph) greater than $3, \beta_{i, i+2}\left(J_{G_{1}, G_{2}}\right)=0$, for all $i$. Moreover, we give a lower bound for the Castelnuovo-Mumford regularity of any binomial edge ideal $J_{G_{1}, G_{2}}$ and hence the ideal of adjacent 2-minors of a generic matrix. We also obtain an upper bound for the regularity of $J_{G_{1}, G_{2}}$, if $G_{1}$ is complete and $G_{2}$ is a closed graph.
\end{abstract}

Keywords: Binomial edge ideal of a pair of graphs, Linear resolutions, Linear relations, Castelnuovo-Mumford regularity

\section{Introduction}

The binomial edge ideal of a graph was introduced in [7], and at about the same time in [13]. Let $G$ be a finite simple graph with vertex set $[n]$ and edge set $E(G)$. Also, let $S=$ $K\left[x_{1}, \ldots, x_{n}, y_{1}, \ldots, y_{n}\right]$ be the polynomial ring over a field $K$. Then the binomial edge ideal of $G$ in $S$, denoted by $J_{G}$, is generated by binomials of the form $f_{i j}=x_{i} y_{j}-x_{j} y_{i}$, where $i<j$ and $\{i, j\} \in E(G)$. This ideal also could be seen as the ideal generated by a collection of 2 -minors of a $(2 \times n)$-matrix whose entries are all indeterminates. In [7], the authors characterized those graphs, which, for certain labeling of their edges, have a

${ }^{*}$ Corresponding author 
quadratic Gröbner basis with respect to the lexicographic order induced by $x_{1}>\cdots>$ $x_{n}>y_{1}>\cdots>y_{n}$. These graphs are called closed graphs. Many of the other algebraic properties of such ideals were studied in [1], [2], [7], [17] and [19]. In [3], the authors introduced the binomial edge ideal of a pair of graphs, as a generalization of the binomial edge ideal of a graph. Let $G_{1}$ be a graph on the vertex set $[m]$ and $G_{2}$ a graph on the vertex set $[n]$, and let $X=\left(x_{i j}\right)$ be an $(m \times n)$-matrix of indeterminates. Let $K[X]$ be the polynomial ring in the variables $x_{i j}$, where $i=1, \ldots, m$ and $j=1, \ldots, n$. Let $e=\{i, j\}$ for some $1 \leqslant i<j \leqslant m$ and $f=\{t, l\}$ for some $1 \leqslant t<l \leqslant n$. To the pair $(e, f)$, the following 2-minor of $X$ is assigned:

$$
p_{e, f}=[i, j \mid t, l]=x_{i t} x_{j l}-x_{i l} x_{j t} .
$$

Then, the ideal

$$
J_{G_{1}, G_{2}}=\left(p_{e, f}: e \in E\left(G_{1}\right), f \in E\left(G_{2}\right)\right)
$$

is called the binomial edge ideal of the pair $\left(G_{1}, G_{2}\right)$. Throughout the paper, by the binomial generators of $J_{G_{1}, G_{2}}$, we mean elements of the form $p_{e, f}$, as above, in $J_{G_{1}, G_{2}}$. If $G_{1}$ is a complete graph, then $J_{G_{1}, G_{2}}$ is the generalized binomial edge ideal attached to $G_{2}$, which studied in [18]. If $G_{1}$ and $G_{2}$ are two paths, then $J_{G_{1}, G_{2}}$ is the ideal of adjacent 2-minors of $X$, which studied for example in [6], [8] and [15]. In [3], those pairs of graphs $\left(G_{1}, G_{2}\right)$ were characterized, for which for a certain labeling of their edges, $J_{G_{1}, G_{2}}$ has a quadratic Gröbner basis with respect to the lexicographic order, induced by $x_{11}>\cdots>x_{1 n}>x_{21}>\cdots>x_{2 n}>\cdots>x_{m 1}>\cdots>x_{m n}$, were characterized. The only pairs with this property, are the pairs $\left(G_{1}, G_{2}\right)$ in which $G_{1}$ is complete and $G_{2}$ is closed, or vice versa. In [3], it was shown that $J_{G_{1}, G_{2}}$ is a radical ideal if and only if either $G_{1}$ or $G_{2}$ is complete. Also, it was proved that $J_{G_{1}, G_{2}}$ is a prime ideal if and only if $G_{1}$ and $G_{1}$ are complete. Moreover, the authors determined all minimal prime ideals of $J_{G_{1}, G_{2}}$, and hence characterized all unmixed binomial edge ideal of pairs of graphs.

In this paper, we study some other algebraic properties and invariants of $J_{G_{1}, G_{2}}$. In particular, when $G_{1}$ is just an edge, we can recover the results of [19] on binomial edge ideals.

Associated to the graph $G$ is also a quadratic squarefree monomial ideal $I(G)=$ $\left(x_{i} x_{j}:\{i, j\} \in E(G)\right)$, in the polynomial ring $R=K\left[x_{1}, \ldots, x_{n}\right]$ over a field $K$, called the edge ideal of $G$. In [4], Fröberg characterized all graphs whose edge ideals have a linear resolution. He showed that $I(G)$ has a linear resolution if and only if the complementary graph $\bar{G}$ is chordal. In [19], the authors determined all graphs whose binomial edge ideals have a linear resolution. They showed that $J_{G}$ has a linear resolution if and only if $J_{G}$ has linear relations, if and only if $G$ is a complete graph. The question arises whether there is a graphical characterization for binomial edge ideals of pairs of graphs to have a linear resolution. In this paper, we give a positive answer to this question. In Section 2, we show that $J_{G_{1}, G_{2}}$ has linear relations only if $G_{1}$ and $G_{2}$ are both complete graphs. Then, we deduce that $J_{G_{1}, G_{2}}$ has a linear resolution, if and only if $G_{1}$ and $G_{2}$ are complete graphs and one of them is just an edge. Also, in this section, we determine some of the Betti numbers of the binomial edge ideal of a pair of graphs. Actually, we show that $\beta_{1,3}\left(J_{G_{1}, G_{2}}\right)=$ 
$2 e\left(G_{1}\right) k_{3}\left(G_{2}\right)+2 e\left(G_{2}\right) k_{3}\left(G_{1}\right)+k_{3}\left(G_{1}\right)\left(p_{3}\left(G_{2}\right)-k_{3}\left(G_{2}\right)\right)+k_{3}\left(G_{2}\right)\left(p_{3}\left(G_{1}\right)-k_{3}\left(G_{1}\right)\right)$, where $e(G), p_{3}(G)$ and $k_{3}(G)$ are the number of edges, 3-paths and 3-cycles of a graph $G$, respectively. Then, we deduce that for all $i>0, \beta_{i, i+2}\left(J_{G_{1}, G_{2}}\right)=0$, for every pairs of graphs with girth greater than 3 . In particular, we deduce that $\beta_{i, i+2}(I)=0$, for all $i>0$, whenever $I$ is the ideal generated by adjacent 2 -minors of the matrix $X$. In addition, we show that if one of $G_{1}$ or $G_{2}$ is a non-complete connected graph, then $\beta_{1,4}\left(J_{G_{1}, G_{2}}\right) \neq 0$.

In Section 3, we give some bounds for the Castelnuovo-Mumford regularity of the binomial edge ideal of a pair of graphs. We give a lower bound for the CastelnuovoMumford regularity of $J_{G_{1}, G_{2}}$, for every pair $\left(G_{1}, G_{2}\right)$ of graphs. Consequently, we give a lower bound for the Castelnuovo-Mumford regularity of the ideal of adjacent 2-minors of an $(m \times n)$ generic matrix. Also, by using an important result of Kalai and Meshulam on the regularity of monomial ideals, we gain an upper bound for the Castelnuovo-Mumford regularity of the binomial edge ideal of a pair of graphs, in which one of the graphs is complete and the other one is closed. Precisely, we show that the regularity of the binomial edge ideal of a pair of graphs $\left(K_{m}, G\right)$, where $K_{m}$ is the complete graph on $[m]$ and $G$ is a closed graph, is less than or equal to $\min \left\{\left(\begin{array}{c}m \\ 2\end{array}\right) c(G), e(G)\right\}+1$, where $c(G)$ is the number of maximal cliques of $G$.

Throughout the paper, we mean by a graph $G$, a simple graph over $n$ vertices, with no isolated vertices. Whenever we say that $G$ is a graph on $[n]$, we mean that the set of vertices of $G$ is $\left\{v_{1}, \ldots, v_{n}\right\}$. Also, by $<$, we mean the lexicographic order induced by $x_{11}>\cdots>x_{1 n}>x_{21}>\cdots>x_{2 n}>\cdots>x_{m 1}>\cdots>x_{m n}$. Moreover, we consider $S$ to be standard graded, unless we mention something else. Non of the results of this paper depends on the characteristic of the field $K$.

\section{The binomial edge ideal of a pair of graphs with linear resolution}

In this section, we study the graded Betti numbers $\beta_{1,3}\left(J_{G_{1}, G_{2}}\right)$ and $\beta_{1,4}\left(J_{G_{1}, G_{2}}\right)$, and we characterize all pairs of graphs $\left(G_{1}, G_{2}\right)$, in which $J_{G_{1}, G_{2}}$ has linear relations. Then, we classify all pairs of graphs $\left(G_{1}, G_{2}\right)$, in which $J_{G_{1}, G_{2}}$ has a linear resolution. The following theorem is one of two main theorems of this section:

Theorem 1. Let $G_{1}$ and $G_{2}$ be two graphs on $[m]$ and $[n]$, respectively. Then the following conditions are equivalent:

(a) $J_{G_{1}, G_{2}}$ has linear relations.

(b) $J_{G_{1}, G_{2}}$ is a toric ideal, and $G_{1}$ and $G_{2}$ are connected.

(c) $G_{1}$ and $G_{2}$ are complete graphs.

In order to prove Theorem 1, we need some facts that we will mention in the following. We denote the number of edges, 3-paths and 3-cycles of a graph $G$, by $e(G), p_{3}(G)$ and $k_{3}(G)$, respectively. In the next result, we determine the first initial graded Betti number of the binomial edge ideal of a graph: 
Theorem 2. Let $G_{1}$ and $G_{2}$ be two graphs on $[m]$ and $[n]$, respectively. Then we have

(a) $\beta_{1,3}\left(J_{G_{1}, G_{2}}\right)=2 e\left(G_{1}\right) k_{3}\left(G_{2}\right)+2 e\left(G_{2}\right) k_{3}\left(G_{1}\right)+k_{3}\left(G_{1}\right)\left(p_{3}\left(G_{2}\right)-k_{3}\left(G_{2}\right)\right)+$ $k_{3}\left(G_{2}\right)\left(p_{3}\left(G_{1}\right)-k_{3}\left(G_{1}\right)\right)$.

(b) $\beta_{1,4}\left(J_{G_{1}, G_{2}}\right) \neq 0$, if either $G_{1}$ or $G_{2}$ is non-complete and connected.

(c) $\beta_{i-1, j}\left(J_{G_{1}, G_{2}}\right)=0$, for $j>2 i$, if $G_{1}$ is closed and $G_{2}$ is complete, or vice versa. In particular, $\beta_{1, j}\left(J_{G_{1}, G_{2}}\right)=0$, for $j \neq 3,4$, if $G_{1}$ is closed and $G_{2}$ is complete, or vice versa.

(d) $\beta_{i, j}\left(J_{G_{1}, G_{2}}\right)=0$, for $j>m n$, if either $G_{1}$ or $G_{2}$ is a complete graph.

Proof. (a) Note that we can consider two different $\mathbb{Z}$-gradings for $S$. One is the standard grading and the other is grading by the weight $\mathbf{w}=(2, \ldots, 2) \in \mathbb{N}^{m n}$. Thus, for every $p, q, \beta_{p, q}\left(J_{G_{1}, G_{2}}\right)$ in the standard grading coincides with $\beta_{p, 2 q}\left(J_{G_{1}, G_{2}}\right)$ in the weighted one. So, here, instead of computing $\beta_{1,3}\left(J_{G_{1}, G_{2}}\right)$ in the standard grading, we will compute $\beta_{1,6}\left(J_{G_{1}, G_{2}}\right)$ in the weighted grading. For every $e=\{i, j\} \in E\left(G_{1}\right)$ and $f=\{k, l\} \in$ $E\left(G_{2}\right)$, we set $p_{i j, k l}:=p_{e, f}$. Suppose that

$$
\cdots \longrightarrow S^{e\left(G_{1}\right) e\left(G_{2}\right)}(-4) \stackrel{\psi}{\longrightarrow} S \longrightarrow S / J_{G_{1}, G_{2}} \longrightarrow 0
$$

is the minimal graded free resolution of $S / J_{G_{1}, G_{2}}$, in which $\psi\left(\epsilon_{i j, k l}\right)=p_{i j, k l}$ such that $\epsilon_{i j, k l}$ is an element of the standard basis of the free $S$-module $S^{e\left(G_{1}\right) e\left(G_{2}\right)}(-4)$. Moreover, $S$ is also $\mathbb{Z}^{m+n}$-multigraded, with $\operatorname{mdeg}\left(x_{i j}\right)=\varepsilon_{i, j+m}$, where $\varepsilon_{i, j+m}$ is the sum of the $i$-th and the $(j+m)$-th canonical basis vectors of $\mathbb{Z}^{m+n}$. So, $\operatorname{mdeg}\left(\epsilon_{i j, k l}\right)=\operatorname{mdeg}\left(p_{i j, k l}\right)=\varepsilon_{i, k+m}+\varepsilon_{j, l+m}$. Let $Z_{1}$ be the relation module of $S / J_{G_{1}, G_{2}}$, and consider a relation $r=\sum g_{i j, k l} \epsilon_{i j, k l}$ of degree 6 (in the weighted grading), that is, an element in $\left(Z_{1}\right)_{6}$. Since $S / J_{G_{1}, G_{2}}$ is $\mathbb{Z}^{m+n}$-graded, it follows that $\left(Z_{1}\right)_{6}$ is also $\mathbb{Z}^{m+n}$-graded, and hence is generated by multihomogeneous elements. Thus we may assume that $r$ is multihomogeneous, say of multidegree $a \in \mathbb{Z}^{m+n}$. Then all nonzero summands $g_{i j, k l} \epsilon_{i j, k l}$ are of multidegree $a$, with $|a|=6$ (here $|a|$ is the sum of the components of $a$ ). Let $g_{i j, k l} \epsilon_{i j, k l} \neq 0$. Then $a=\operatorname{mdeg}\left(g_{i j, k l}\right)+\operatorname{mdeg}\left(\epsilon_{i j, k l}\right)=\operatorname{mdeg}\left(g_{i j, k l}\right)+\varepsilon_{i, k+m}+\varepsilon_{j, l+m}$. Therefore, $\operatorname{mdeg}\left(g_{i j, k l}\right)=\varepsilon_{s, t+m}$ for some $s, t$. If $s=i$ and $t=k$ or $l$, then $a=2 \varepsilon_{i, k+m}+\varepsilon_{j, l+m}$ or $\varepsilon_{i, l+m}+\varepsilon_{i, k+m}+\varepsilon_{j, l+m}$, and hence there is only one summand in $r$ with this multidregree and $r \notin Z_{1}$, a contradiction. Similarly, if $s=j$ and $t=k$ or $l$, then $r \notin Z_{1}$, a contradiction. So, it remains to consider the following cases:

Case (1). Suppose that $s=i$ and $t \neq k, l$. Let $t<k<l$. Then, $a=\varepsilon_{i, t+m}+\varepsilon_{i, k+m}+\varepsilon_{j, l+m}$. So, $r$ has exactly three summands and hence $r=g_{i j, t k} \epsilon_{i j, t k}+g_{i j, t l} \epsilon_{i j, t l}+g_{i j, k l} \epsilon_{i j, k l}$. But, it happens if and only if $G_{2}$ contains a 3 -cycle over the vertices $t, k$ and $l$. Thus $r$ is a relation of the ideal $\left(p_{i j, t k}, p_{i j, t l}, p_{i j, k l}\right)$, which is the ideal of 2-minors of the matrix $\left[\begin{array}{lll}x_{i t} & x_{i k} & x_{i l} \\ x_{j t} & x_{j k} & x_{j l}\end{array}\right]$. So, the generating relations 
are $x_{i l} \epsilon_{i j, t k}-x_{i k} \epsilon_{i j, t l}+x_{i t} \epsilon_{i j, k l}$ and $x_{j l} \epsilon_{i j, t k}-x_{j k} \epsilon_{i j, t l}+x_{j t} \epsilon_{i j, k l}$, by Hilbert-Burch theorem. But, multidegree of the latter is not equal to $a$. Hence, in this multidegree, we just consider $x_{i l} \epsilon_{i j, t k}-x_{i k} \epsilon_{i j, t l}+x_{i t} \epsilon_{i j, k l}$. Therefore, in this case, we obtain $e\left(G_{1}\right) k_{3}\left(G_{2}\right)$ elements in $\left(Z_{1}\right)_{6}$.

Case (2). Suppose that $s=j$ and $t \neq k, l$. Let $t<k<l$. Then, $a=\varepsilon_{j, t+m}+\varepsilon_{i, k+m}+\varepsilon_{j, l+m}$. So, $r$ has exactly three summands and hence $r=g_{i j, t k} \epsilon_{i j, t k}+g_{i j, t l} \epsilon_{i j, t l}+g_{i j, k l} \epsilon_{i j, k l}$. But, it happens if and only if $G_{2}$ contains a 3 -cycle over the vertices $t, k$ and $l$. By repeating the discussion in Case (1), we obtain that $x_{j l} \epsilon_{i j, t k}-x_{j k} \epsilon_{i j, t l}+x_{j t} \epsilon_{i j, k l}$ is the only possible generating relation in this case. Thus, in this case, we get $e\left(G_{1}\right) k_{3}\left(G_{2}\right)$ elements in $\left(Z_{1}\right)_{6}$.

Case (3). Suppose that $s \neq i, j$ and $t=k$. Let $s<i<j$. Then, $a=\varepsilon_{s, k+m}+\varepsilon_{i, k+m}+\varepsilon_{j, l+m}$. So, $r$ has exactly three summands and hence $r=g_{s i, k l} \epsilon_{s i, k l}+g_{s j, k l} \epsilon_{s j, k l}+g_{i j, k l} \epsilon_{i j, k l}$. But, it happens if and only if $G_{1}$ contains a 3 -cycle over the vertices $s, i$ and $j$. Thus $r$ is a relation of the ideal $\left(p_{s i, k l}, p_{s j, k l}, p_{i j, k l}\right)$, which is the ideal of 2-minors of the matrix $\left[\begin{array}{ccc}x_{s k} & x_{i k} & x_{j k} \\ x_{s l} & x_{i l} & x_{j l}\end{array}\right]$. So, the generating relations are $x_{j k} \epsilon_{s i, k l}-x_{i k} \epsilon_{s j, k l}+x_{s k} \epsilon_{i j, k l}$ and $x_{j l} \epsilon_{i j, t k}-x_{i l} \epsilon_{i j, t l}+x_{s l} \epsilon_{i j, k l}$, by Hilbert-Burch theorem. But, multidegree of the latter is not equal to $a$. Hence, in this multidegree, we just consider $x_{j k} \epsilon_{s i, k l}-x_{i k} \epsilon_{s j, k l}+x_{s k} \epsilon_{i j, k l}$. Therefore, in this case, we obtain $e\left(G_{2}\right) k_{3}\left(G_{1}\right)$ elements in $\left(Z_{1}\right)_{6}$.

Case (4). Suppose that $s \neq i, j$ and $t=l$. Let $s<i<j$. Then, $a=\varepsilon_{s, l+m}+\varepsilon_{i, k+m}+\varepsilon_{j, l+m}$. So, $r$ has exactly three summands and hence $r=g_{s i, k l} \epsilon_{s i, k l}+g_{s j, k l} \epsilon_{s j, k l}+g_{i j, k l} \epsilon_{i j, k l}$. But, it happens if and only if $G_{1}$ contains a 3 -cycle over the vertices $s, i$ and $j$. By repeating the discussion in Case (3), we obtain that $x_{j l} \epsilon_{i j, t k}-x_{i l} \epsilon_{i j, t l}+x_{s l} \epsilon_{i j, k l}$ is the only possible generating relation in this case. Thus, in this case, we get $e\left(G_{2}\right) k_{3}\left(G_{1}\right)$ elements in $\left(Z_{1}\right)_{6}$.

Case (5). Suppose that $s \neq i, j$ and $t \neq k, l$. Let $s<i<j$ and $t<k<l$. Then, $a=\varepsilon_{s, t+m}+\varepsilon_{i, k+m}+\varepsilon_{j, l+m}$. Thus, we have $r=g_{s i, t k} \epsilon_{s i, t k}+g_{s i, t l} \epsilon_{s i, t l}+$ $g_{s i, k l} \epsilon_{s i, k l}+g_{s j, t k} \epsilon_{s j, t k}+g_{s j, t l} \epsilon_{s j, t l}+g_{s j, k l} \epsilon_{s j, k l}+g_{i j, t k} \epsilon_{i j, t k}+g_{i j, t l} \epsilon_{i j, t l}+g_{i j, k l} \epsilon_{i j, k l}$. Since $\operatorname{mdeg}(r)=a=\varepsilon_{s, t+m}+\varepsilon_{i, k+m}+\varepsilon_{j, l+m}$, we have $r=c_{1} x_{j l} \epsilon_{s i, t k}+c_{2} x_{j k} \epsilon_{s i, t l}+c_{3} x_{j t} \epsilon_{s i, k l}+$ $c_{4} x_{i l} \epsilon_{s j, t k}+c_{5} x_{i k} \epsilon_{s j, t l}+c_{6} x_{i t} \epsilon_{s j, k l}+c_{7} x_{s l} \epsilon_{i j, t k}+c_{8} x_{s k} \epsilon_{i j, t l}+c_{9} x_{s t} \epsilon_{i j, k l}$, where $c_{1}, \ldots, c_{9} \in K$. By easy computations, we have that the generating relations in this case, are exactly correspond to the solution space of the homogeneous system of equations $c_{1}+c_{5}+c_{9}=0$, $-c_{1}+c_{6}+c_{8}=0, c_{2}+c_{4}-c_{9}=0, c_{2}+c_{6}-c_{7}=0, c_{3}-c_{4}-c_{8}=0$ and $c_{3}+c_{5}+c_{7}=0$, whose dimension is 4 . Thus, the generating relations are as follows: $r_{1}=x_{j l} \epsilon_{s i, t k}-x_{j k} \epsilon_{s i, t l}+x_{j t} \epsilon_{s i, k l}+x_{i l} \epsilon_{s j, t k}-x_{i k} \epsilon_{s j, t l}+x_{i t} \epsilon_{s j, k l}$, where the vertices $i, s, j$ make a 3-path with edges $\{s, i\}$ and $\{s, j\}$ in $G_{1}$, and the vertices $t, k, l$ induce a 3-cycle in $G_{2} ; r_{2}=x_{s l} \epsilon_{i j, t k}-x_{s k} \epsilon_{i j, t l}+x_{s t} \epsilon_{i j, k l}+x_{i l} \epsilon_{s j, t k}-x_{i k} \epsilon_{s j, t l}+x_{i t} \epsilon_{s j, k l}$, where the vertices $i, s, j$ make a 3-path with edges $\{s, j\}$ and $\{i, j\}$ in $G_{1}$, and the vertices $t, k, l$ induce a 
3-cycle in $G_{2} ; r_{3}=x_{j l} \epsilon_{s i, t k}-x_{i l} \epsilon_{s j, t k}+x_{s l} \epsilon_{i j, t k}+x_{j k} \epsilon_{s i, t l}-x_{i k} \epsilon_{s j, t l}+x_{s k} \epsilon_{i j, t l}$, where the vertices $i, s, j$ induce a 3 -cycle in $G_{1}$, and the vertices $t, k, l$ make a 3 -path with edges $\{t, k\}$ and $\{t, l\}$ in $G_{2} ; r_{4}=x_{j k} \epsilon_{s i, t l}-x_{i k} \epsilon_{s j, t l}+x_{s k} \epsilon_{i j, t l}+x_{j t} \epsilon_{s i, k l}-x_{i t} \epsilon_{s j, k l}+x_{s t} \epsilon_{i j, k l}$, where the vertices $i, s, j$ induce a 3 -cycle in $G_{1}$, and the vertices $t, k, l$ make a 3 -path with edges $\{t, l\}$ and $\{k, l\}$ in $G_{2}$. Note that if the vertices $i, s, j$ do not induce any cycles in $G_{1}$, then just one of the elements $r_{1}$ and $r_{2}$ could appear. Similarly, if the vertices $t, k, l$ do not induce any cycles in $G_{2}$, then just one of the elements $r_{3}$ and $r_{4}$ could appear. Therefore, in this case, we have $k_{3}\left(G_{2}\right)\left(p_{3}\left(G_{1}\right)-k_{3}\left(G_{1}\right)\right)$ elements in $\left(Z_{1}\right)_{6}$, regarding $r_{1}$ and $r_{2}$, and also $k_{3}\left(G_{1}\right)\left(p_{3}\left(G_{2}\right)-k_{3}\left(G_{2}\right)\right)$ elements in $\left(Z_{1}\right)_{6}$, regarding $r_{3}$ and $r_{4}$.

Comparing the multidegrees in these 5 cases, we obtain that the minimal generating relations of degree 6 are of the above forms, and hence the result follows.

(b) Without loss of generality, we may assume that $G_{2}$ is connected and non-complete. Thus, there exist 3 vertices $k, t, l$ in $[n]$ with $k<t<l$ which induce a 3-path in $G_{2}$ with edges $\{k, t\}$ and $\{t, l\}$. Suppose that $\{i, j\}$ is an edge in $G_{1}$. Let $\gamma:=p_{i j, k t} \epsilon_{i j, t l}-p_{i j, t l} \epsilon_{i j, k t}$. Clearly, $\gamma \in Z_{1}$ and $\operatorname{deg}(\gamma)=4$ in the standard grading. We show that $\gamma$ is a minimal relation. Then we have $\beta_{1,4}\left(J_{G_{1}, G_{2}}\right)>0$. Note that $\operatorname{deg}_{\mathbf{w}}(\gamma)=8$ (with the weighted grading mentioned in part (a)), and using $\mathbb{Z}^{m+n}$-grading introduced in part (a), we have $\operatorname{mdeg}(\gamma)=\varepsilon_{i, k+m}+\varepsilon_{j, t+m}+\varepsilon_{i, t+m}+\varepsilon_{j, l+m}$. If $\gamma$ is not a minimal relation, then it must be reduced by elements of $\left(Z_{1}\right)_{6}$. By comparing the multidegrees of $\gamma$ and the generating relations in $\left(Z_{1}\right)_{6}$, one obtains that non of the generating relations of the form of Case (3), Case (4) and Case (5) could occur in the expression of $\gamma$ in terms of the elements of $\left(Z_{1}\right)_{6}$. If the generating relations of the form of Case (1) and Case (2) occur in that expression of $\gamma$, then the vertices $k, t, l$ induce a 3 -cycle in $G_{2}$, which is a contradiction, since $\{k, l\}$ is not an edge of $G_{2}$.

(c) Suppose that $G_{1}$ is complete and $G_{2}$ is closed. Then we have $\operatorname{in}_{<}\left(J_{G_{1}, G_{2}}\right)=\left(x_{i k} x_{j l}: i<j, k<l,\{i, j\} \in E\left(G_{1}\right),\{k, l\} \in E\left(G_{2}\right)\right)$, by [3, Theorem 1.3]. Thus, it can be seen as the edge ideal of an $n$-partite graph over the vertex set $V=\bigcup_{p=1}^{n} V_{p}$, where $V_{p}=\left\{x_{1 p}, \ldots, x_{m p}\right\}$, for all $p=1, \ldots, n$. We denote this $n$-partite graph by $\operatorname{in}_{<}\left(G_{1}, G_{2}\right)$. So, we have $\operatorname{in}_{<}\left(J_{G_{1}, G_{2}}\right)=I\left(\operatorname{in}_{<}\left(G_{1}, G_{2}\right)\right)$. But, $\beta_{i-1, j}\left(I\left(\operatorname{in}_{<}\left(G_{1}, G_{2}\right)\right)\right)=0$, for all $j>2 i$, by [10, Lemma 2.2]. On the other hand, we have $\beta_{i-1, j}\left(J_{G_{1}, G_{2}}\right) \leqslant \beta_{i-1, j}\left(\operatorname{in}_{<}\left(J_{G_{1}, G_{2}}\right)\right)$, for all $i, j$, by [5, Corollary 3.3.3]. So, if $G_{1}$ is complete and $G_{2}$ is closed, then $\beta_{i-1, j}\left(J_{G_{1}, G_{2}}\right)=0$, for all $j>2 i$.

(d) By [3, Theorem 1.2], in $\operatorname{in}_{<}\left(J_{G_{1}, G_{2}}\right)$ is a squarefree monomial ideal in $S$. Thus, the result follows by Hochster's formula, since $\beta_{i, j}\left(J_{G_{1}, G_{2}}\right) \leqslant \beta_{i, j}\left(\operatorname{in}_{<}\left(J_{G_{1}, G_{2}}\right)\right)$, for all $i, j$.

Notice that, by setting $G_{1}=K_{2}$, Theorem 2 yields all parts of [19, Theorem 2.2].

The length of any shortest cycle (if any) in a graph $G$ is called the girth of $G$. The girth of acyclic graphs, i.e. graphs with no cycle, is considered as infinity. 
Corollary 3. If $G_{1}$ and $G_{2}$ are graphs with girth greater than 3, then $\beta_{i, i+2}\left(J_{G_{1}, G_{2}}\right)=0$, for all $i>0$. In particular, if $G_{1}$ and $G_{2}$ are bipartite graphs, one has $\beta_{i, i+2}\left(J_{G_{1}, G_{2}}\right)=0$, for all $i>0$.

A $(2 \times 2)$ adjacent minor of $X$ is the determinant of a submatrix with row indices $i, i+1$ and column indices $j, j+1$. We call the ideal generated by all of the $(2 \times 2)$ adjacent minors of $X$, the ideal of adjacent 2-minors of $X$.

Corollary 4. Let I be the ideal of adjacent 2-minors of an $(m \times n)$ generic matrix. Then $\beta_{1,4}(I) \neq 0$, and $\beta_{i, i+2}(I)=0$, for all $i>0$.

Proof. It is enough to note that $I=J_{P_{m}, P_{n}}$.

Applying Theorem 2, part (a), we gain the following:

Corollary 5. Let $m, n \geqslant 3$ and $t \geqslant 4$. Then

(a) $\beta_{1,3}\left(J_{K_{m}, K_{n}}\right)=2\left(\left(\begin{array}{c}m \\ 3\end{array}\right)\left(\begin{array}{c}n+1 \\ 3\end{array}\right)+\left(\begin{array}{c}n \\ 3\end{array}\right)\left(\begin{array}{c}m+1 \\ 3\end{array}\right)\right)$.

(b) $\beta_{1,3}\left(J_{K_{m}, C_{t}}\right)=3 t\left(\begin{array}{c}m \\ 3\end{array}\right)$, where $C_{t}$ is a cycle over $t$ vertices.

(c) $\beta_{1,3}\left(J_{K_{m}, T}\right)=\left(2 n+p_{3}(T)-2\right)\left(\begin{array}{c}m \\ 3\end{array}\right)$, where $T$ is a tree over $n$ vertices. In particular, $\beta_{1,3}\left(J_{K_{m}, P_{n}}\right)=(3 n-4)\left(\begin{array}{c}m \\ 3\end{array}\right)$.

Remark 6. If $G_{1}$ is a closed graph and $G_{2}$ is complete, or vice versa, we apply consecutive cancellations to show that $\beta_{1,3}\left(J_{G_{1}, G_{2}}\right)=\beta_{1,3}\left(\operatorname{in}_{<}\left(J_{G_{1}, G_{2}}\right)\right)$. Actually, we have $\beta_{0,3}\left(J_{G_{1}, G_{2}}\right)=\beta_{0,3}\left(\operatorname{in}_{<}\left(J_{G_{1}, G_{2}}\right)\right)=0$ and $\beta_{2,3}\left(J_{G_{1}, G_{2}}\right)=\beta_{2,3}\left(\operatorname{in}_{<}\left(J_{G_{1}, G_{2}}\right)\right)=0$, by minimality of the free resolutions. On the other hand, by [16, Theorem 22.12], the sequence of graded Betti numbers of $J_{G_{1}, G_{2}}$ is obtained from the sequence of graded Betti numbers of $\operatorname{in}_{<}\left(J_{G_{1}, G_{2}}\right)$ by consecutive cancellations. So, we have $\beta_{1,3}\left(J_{G_{1}, G_{2}}\right)=\beta_{1,3}\left(\operatorname{in}_{<}\left(J_{G_{1}, G_{2}}\right)\right)$. A sequence $q_{i, j}$ of numbers is said to be obtained from a sequence $p_{i, j}$ by a consecutive cancellation if there exist indices $s$ and $r$ such that $q_{s, r}=p_{s, r}-1, q_{s+1, r}=p_{s+1, r}-1$ and $q_{i, j}=p_{i, j}$ for all other values of $i, j$.

Recall that a homogeneous ideal $I$ whose generators all have degree $d$ is said to have a $d$-linear resolution (or simply linear resolution) if for all $i \geqslant 0, \beta_{i, j}(I)=0$ for all $j \neq i+d$. Also, if $\beta_{1, j}(I)=0$ for all $j \neq d+1$, then we say that $I$ has linear relations.

Now, we are ready to prove Theorem 1 .

Proof of Theorem 1. (a) $\Rightarrow$ (c): Suppose that $J_{G_{1}, G_{2}}$ has linear relations. Thus, $\beta_{1, j}\left(J_{G_{1}, G_{2}}\right)=0$, for all $j>3$. In particular, $\beta_{1,4}\left(J_{G_{1}, G_{2}}\right)=0$. So that $G_{1}$ and $G_{2}$ are both connected, since if one of them, say $G_{1}$, has connected components $H_{1}, \ldots, H_{c}$, then the minimal graded free resolution of $S / J_{G_{1}, G_{2}}$ is the tensor product of those of $S / J_{G_{1}, H_{1}}, \ldots, S / J_{G_{1}, H_{c}}$, and hence $\beta_{1,4}\left(J_{G_{1}, G_{2}}\right) \geqslant \sum_{1 \leqslant i<j \leqslant c} \beta_{0,2}\left(J_{G_{1}, H_{i}}\right) \beta_{0,2}\left(J_{G_{1}, H_{j}}\right)>0$. Therefore, $G_{1}$ and $G_{2}$ are both complete graphs, by Theorem 2, part (b). 
(c) $\Rightarrow$ (a): Suppose that $G_{1}$ and $G_{2}$ are complete graphs. Then $J_{G_{1}, G_{2}}=I_{2}(X)$ is the ideal of 2-minors of $X$, the $(m \times n)$-matrix of indeterminates. Thus, by Kurano's theorem, $J_{G_{1}, G_{2}}$ has linear relations (see [11]).

(b) $\Rightarrow$ (c): Suppose that $J_{G_{1}, G_{2}}$ is a toric ideal. Then, it is a prime ideal. So, $G_{1}$ and $G_{2}$ are both complete graphs, by [3, Corollary 2.2].

(c) $\Rightarrow$ (b): Let $G_{1}$ and $G_{2}$ be complete graphs. Then, by [21, Proposition 9.1.2],

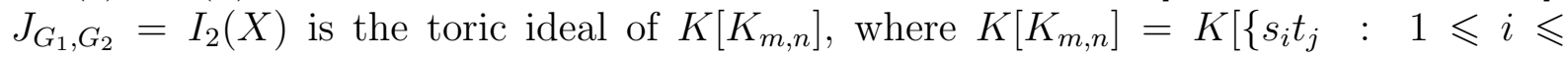
$m, 1 \leqslant j \leqslant n\}]$ and $K_{m, n}$ is the complete bipartite graph over the set of vertices $\left\{s_{1}, \ldots, s_{m}, t_{1}, \ldots, t_{n}\right\}$. More precisely, $J_{G_{1}, G_{2}}$ is the kernel of the graded homomorphism of $K$-algebras

$$
\varphi: K\left[\left\{x_{i j}: 1 \leqslant i \leqslant m 1 \leqslant j \leqslant n\right\}\right] \longrightarrow K\left[K_{m, n}\right],
$$

in which $\varphi\left(x_{i j}\right)=s_{i} t_{j}$, for all $i, j$.

Remark 7. By [19, Theorem 2.1], when $G_{1}=K_{2}$, we have that $J_{G_{1}, G_{2}}$ has linear relations if and only if $\operatorname{in}_{<}\left(J_{G_{1}, G_{2}}\right)$ does. But this is not true, in general, that is when $G_{1}$ is not an edge. For example, computations by $\mathrm{CoCo} A$ show that $\beta_{1,4}\left(\mathrm{in}_{<}\left(J_{K_{3}, K_{3}}\right)\right)=1$, and hence $\operatorname{in}_{<}\left(J_{K_{3}, K_{3}}\right)$ does not have linear relations.

Let $B$ be a $K$-algebra and $A$ a $K$-subalgebra of $B$. Recall that $A$ is called an algebra retract of $B$, if there exists a surjective $K$-algebra homomorphism $\pi: B \rightarrow A$ whose composition with the inclusion map $A \rightarrow B$ is the identity on $A$.

Let $G_{1}$ and $G_{2}$ be graphs on $[m]$ and $[n]$, and let $H_{1}$ and $H_{2}$ be subgraphs of $G_{1}$ and $G_{2}$ over $m_{1} \leqslant m$ and $n_{1} \leqslant n$ vertices, respectively. So, one could consider an $m_{1} \times n_{1}$ submatrix $Y$ of $X$, correspond to the vertices of $H_{1}$ and $H_{2}$. Here, we also use $Y$ to denote the set of variables appeared in the matrix $Y$. So that the binomial generators of $J_{H_{1}, H_{2}}$ are contained in the ring $K[Y]$. With these notations, we have the following proposition:

Proposition 8. Let $G_{1}$ and $G_{2}$ be two graphs over $[m]$ and $[n]$, respectively. If $H_{1}$ and $\mathrm{H}_{2}$ are induced subgraphs of $G_{1}$ and $G_{2}$, respectively, then we have

(a) $\beta_{i, j}^{K[Y]}\left(J_{H_{1}, H_{2}}\right) \leqslant \beta_{i, j}^{K[X]}\left(J_{G_{1}, G_{2}}\right)$, for all $i, j$.

(b) $\operatorname{reg}_{K[Y]}\left(J_{H_{1}, H_{2}}\right) \leqslant \operatorname{reg}_{K[X]}\left(J_{G_{1}, G_{2}}\right)$.

(c) $\operatorname{pd}_{K[Y]}\left(J_{H_{1}, H_{2}}\right) \leqslant \operatorname{pd}_{K[X]}\left(J_{G_{1}, G_{2}}\right)$.

Proof. (a) Let $H_{1}$ and $H_{2}$ be induced subgraphs of $G_{1}$ and $G_{2}$ over $m_{1} \leqslant m$ and $n_{1} \leqslant n$ vertices, respectively. So, one could consider an $m_{1} \times n_{1}$ submatrix $Y$ of $X$, correspond to the vertices of $H_{1}$ and $H_{2}$. Note that $J_{H_{1}, H_{2}}$ is an ideal of $K[X]$ whose binomial generators are in $K[Y]$. By $J_{H_{1}, H_{2}} K[Y]$, we mean an ideal of $K[Y]$, whose generators are the same as $J_{H_{1}, H_{2}}$ (as an ideal of $K[X]$ ). We have $J_{G_{1}, G_{2}} \cap K[Y]=J_{H_{1}, H_{2}} K[Y]$. Because, obviously, $J_{H_{1}, H_{2}} K[Y] \subseteq J_{G_{1}, G_{2}} \cap K[Y]$. For the other containment, let $f \in J_{G_{1}, G_{2}} \cap K[Y]$. So, $f=\sum_{s=1}^{t} r_{s} p_{s}$, for some binomial generators $p_{s}$ of $J_{G_{1}, G_{2}}$ and $r_{s} \in K[X]$, where $s=1, \ldots, t$. Now, it is enough to set all variables $x_{i j}$ in $X$, which do not belong to $Y$, equal to zero in $f=\sum_{s=1}^{t} r_{s} p_{s}$. The left hand side of this equality does not change, since $f \in K[Y]$. But, in the right hand side, if $p_{i}=x_{j k} x_{q l}-x_{j l} x_{q k} \notin K[Y]$, for some $i$, then one 
of the variables appeared in $p_{i}$ does not belong to $Y$, say $x_{j k} \notin Y$. So, $j \notin\left[m_{1}\right]$ or $k \notin\left[n_{1}\right]$. Hence, $x_{j l} \notin Y$ or $x_{q k} \notin Y$. So, after substituting desired variables by zero, $p_{i}$ will be omitted in the expression of $f$. Thus, we get $f=\sum_{i=1}^{t^{\prime}} r_{s_{i}}^{\prime} p_{s_{i}}$, where $r_{s_{i}}^{\prime}$ 's are obtained by putting zero instead of variables of $X \backslash Y$ in $r_{s}$ 's, and $p_{s_{i}}$ 's belong to $K[Y]$. Since $H_{1}$ and $H_{2}$ are induced subgraphs of $G_{1}$ and $G_{2}, p_{s_{i}}$ 's belong to $J_{H_{1}, H_{2}} K[Y]$, and hence $f \in J_{H_{1}, H_{2}} K[Y]$. Now, set $A=K[Y] / J_{H_{1}, H_{2}} K[Y]$ and $B=K[X] / J_{G_{1}, G_{2}}$. Thus, $A$ is a $K$ subalgebra of $B$. Let $\pi: B \rightarrow A$ be the epimorphism induced by setting all variables $x_{i j}$ in $X$, which do not belong to $Y$, equal to zero. So, we have the maps $A \hookrightarrow B \stackrel{\pi}{\rightarrow} A$ whose composition is the identity on $A$. Hence, $A$ is an algebra retract of $B$. Now, applying [14, Corollary 2.8], the result follows. Parts (b) and (c) follow immediately from (a).

Corollary 9. Let $G_{1}$ and $G_{2}$ be two graphs over $[m]$ and $[n]$, respectively, and let $S_{1}=$ $\left[x_{i}, y_{i}: 1 \leqslant i \leqslant m\right]$ and $S_{2}=\left[x_{i}, y_{i}: 1 \leqslant i \leqslant n\right]$. Then we have

(a) $\beta_{i j}^{K[X]}\left(J_{G_{1}, G_{2}}\right) \geqslant \max \left\{\beta_{i j}^{S_{1}}\left(J_{G_{1}}\right), \beta_{i j}^{S_{2}}\left(J_{G_{2}}\right)\right\}$, for all $i, j$.

(b) $\operatorname{reg}_{K[X]}\left(J_{G_{1}, G_{2}}\right) \geqslant \max \left\{\operatorname{reg}_{S_{1}}\left(J_{G_{1}}\right), \operatorname{reg}_{S_{2}}\left(J_{G_{2}}\right)\right\}$.

(c) $\operatorname{pd}_{K[X]}\left(J_{G_{1}, G_{2}}\right) \geqslant \max \left\{\operatorname{pd}_{S_{1}}\left(J_{G_{1}}\right), \operatorname{pd}_{S_{2}}\left(J_{G_{2}}\right)\right\}$.

Proof. since $G_{1}$ and $G_{2}$ contain at least an edge, by Proposition 8, we have that $\beta_{i j}^{K\left[Y_{1}\right]}\left(J_{G_{1}, K_{2}}\right) \leqslant \beta_{i j}^{K[X]}\left(J_{G_{1}, G_{2}}\right)$ and $\beta_{i j}^{K\left[Y_{2}\right]}\left(J_{K_{2}, G_{2}}\right) \leqslant \beta_{i j}^{K[X]}\left(J_{G_{1}, G_{2}}\right)$, for all $i, j$, where $Y_{1}$ and $Y_{2}$ are appropriate subsets of $X$. But, obviously, $\beta_{i j}^{K\left[Y_{1}\right]}\left(J_{G_{1}, K_{2}}\right)=\beta_{i j}^{S_{1}}\left(J_{G_{1}}\right)$ and $\beta_{i j}^{K\left[Y_{2}\right]}\left(J_{K_{2}, G_{2}}\right)=\beta_{i j}^{S_{2}}\left(J_{G_{2}}\right)$. So, we get part (a). Parts (b) and (c) follow immediately from (a).

Now, we go to the second main result of this section:

Theorem 10. Let $G_{1}$ and $G_{2}$ be two graphs over $[m]$ and $[n]$, respectively. Then $J_{G_{1}, G_{2}}$ has a linear resolution if and only if $G_{1}$ and $G_{2}$ are complete graphs, and $m=2$ or $n=2$.

Proof. If $G_{1}$ and $G_{2}$ are complete graphs such that one of them is an edge, then $J_{G_{1}, G_{2}}$ has a linear resolution, by [19, Theorem 2.1]. Conversely, suppose that $J_{G_{1}, G_{2}}$ has a linear resolution. So, it has linear relations, and hence $G_{1}$ and $G_{2}$ are both complete graphs, on $[m]$ and $[n]$, respectively, by Theorem 1 . Suppose on the contrary that $m, n \geqslant 3$. Then, both of $G_{1}$ and $G_{2}$ have an induced 3-cycle. Thus, the graded Betti numbers of $J_{G_{1}, G_{2}}$ is greater than or equal to the graded Betti numbers of $J_{K_{3}, K_{3}}$, by Proposition 8 . On the other hand, by [20, Theorem 5.4.6], $S / J_{K_{3}, K_{3}}$ is Gorenstein, and hence its minimal graded free resolution is symmetric. So that $J_{K_{3}, K_{3}}$ has no linear resolution, since it is generated by quadratic forms. Hence, $J_{G_{1}, G_{2}}$ has no linear resolution as well, a contradiction. So, we have $m=2$ or $n=2$.

\section{The Castelnuovo-Mumford regularity of the bino- mial edge ideal of a pair of graphs}

In this section, we study the Castelnuovo-Mumford regularity (or regularity, for short,) of the binomial edge ideal of a pair of graphs. Indeed, we give a lower bound for the 
regularity of the binomial edge ideal of an arbitrary pair of graphs. Consequently, we obtain a lower bound for the ideals of adjacent 2-minors. Also, we obtain an upper bound for the regularity of the binomial edge ideal of a pair of graphs $\left(K_{m}, G\right)$, in which $G$ is a closed graph. In order to prove the main theorem of this section, we need some facts which we will mention in the sequel.

Notice that if $G$ is a closed graph, then we have $\operatorname{in}_{<}\left(J_{G}\right)=\left(x_{i} y_{j}: i<j,\left\{v_{i}, v_{j}\right\} \in\right.$ $E(G))$. Thus, it can be seen as the edge ideal of a bipartite graph over the vertex set $V=\left\{x_{1}, \ldots, x_{n}, y_{1}, \ldots, y_{n}\right\}$. We denote this bipartite graph by $\operatorname{in}_{<}(G)$. So, we have $\operatorname{in}_{<}\left(J_{G}\right)=I\left(\operatorname{in}_{<}(G)\right)$ (see also [19, Theorem 2.2, part (c)]). Moreover, as we mentioned in the proof of Theorem 2, if $G_{1}$ is complete and $G_{2}$ is closed, then we have $\operatorname{in}_{<}\left(J_{G_{1}, G_{2}}\right)=$ $\left(x_{i k} x_{j l}: i<j, k<l,\{i, j\} \in E\left(G_{1}\right),\{k, l\} \in E\left(G_{2}\right)\right)$, by [3, Theorem 1.3]. So that it can be seen as the edge ideal of an $n$-partite graph over the vertex set $V=\bigcup_{p=1}^{n} V_{p}$, where $V_{p}=\left\{x_{1 p}, \ldots, x_{m p}\right\}$, for all $p=1, \ldots, n$. We denote this $n$-partite graph by $\operatorname{in}_{<}\left(G_{1}, G_{2}\right)$. Thus, we have $\operatorname{in}_{<}\left(J_{G_{1}, G_{2}}\right)=I\left(\operatorname{in}_{<}\left(G_{1}, G_{2}\right)\right)$.

A graph $G$ is called chordal if each induced cycle in $G$ has length 3, and $G$ is called co-chordal if the complementary graph $\bar{G}$ is chordal. The co-chordal cover number of a graph $G$, which is denoted by $\operatorname{cochord}(G)$, is the minimum number of subgraphs $H_{1}, \ldots, H_{s}$ of $G$ such that every $H_{i}$ is cochordal and $\bigcup_{i=1}^{s} E\left(H_{i}\right)=E(G)$.

In [22], Woodroofe posed an upper bound for the regularity of the edge ideal of a graph:

Theorem 11. [22, Theorem 11] For any graph $G$, we have $\operatorname{reg}(I(G)) \leqslant \operatorname{cochord}(G)+1$.

The following theorem is a special case of the result proved by Kalai and Meshulam. Their result is on simplicial complexes, in general.

Theorem 12. [9, Theorem 1.2] If $G_{1}, \ldots, G_{s}$ are graphs on the same vertex set, then $\operatorname{reg}\left(S / I\left(\bigcup_{i=1}^{s} G_{i}\right)\right) \leqslant \sum_{i=1}^{s} \operatorname{reg}\left(S / I\left(G_{i}\right)\right)$.

We denote by $c(G)$, the number of maximal cliques of the graph $G$. Here, we mean by a maximal clique of a graph $G$, an induced subgraph of $G$ which is a complete graph and is also maximal with this property. Now, we are ready to prove the main theorem of this section:

Theorem 13. Let $G$ be a closed graph on $[n]$ and $m, n \geqslant 2$. Then we have

$$
\operatorname{reg}\left(J_{K_{m}, G}\right) \leqslant \min \left\{\left(\begin{array}{c}
m \\
2
\end{array}\right) c(G), e(G)\right\}+1
$$

Proof. Since $G$ is closed, we have $\operatorname{in}_{<}\left(J_{G}\right)=I\left(\operatorname{in}_{<}(G)\right)$ and $\operatorname{in}_{<}\left(J_{K_{m}, G}\right)=I\left(\operatorname{in}_{<}\left(K_{m}, G\right)\right)$, as we mentioned above. Note that $\operatorname{in}_{<}\left(K_{m}, G\right)$ could be seen as a multipartite graph in the following two ways:

(1) Consider $\operatorname{in}_{<}\left(K_{m}, G\right)$ as an $n$-partite graph over the vertex set $V=\bigcup_{p=1}^{n} V_{p}$, where $V_{p}=\left\{x_{1 p}, \ldots, x_{m p}\right\}$, for all $p=1, \ldots, n$. It can be easily checked that for every $i, j$ with $1 \leqslant i<j \leqslant n$, the induced subgraph of $\operatorname{in}_{<}\left(K_{m}, G\right)$ on $V_{i, j}:=V_{i} \cup V_{j}$ is isomorphic to 
$\operatorname{in}_{<}\left(K_{m}\right)$. On the other hand, we have that $\operatorname{in}_{<}\left(K_{m}, G\right)=\bigcup_{\{i, j\} \in E(G)}\left(\operatorname{in}_{<}\left(K_{m}, G\right)\right)_{V_{i j}}$. Thus, by Theorem 12, we obtain

$$
\operatorname{reg}\left(S / I\left(\operatorname{in}_{<}\left(K_{m}, G\right)\right)\right) \leqslant e(G) \operatorname{reg}\left(S / I\left(\operatorname{in}_{<}\left(K_{m}\right)\right)\right)=e(G)
$$

where the last equality holds, since $\operatorname{reg}\left(S / I\left(\operatorname{in}_{<}\left(K_{m}\right)\right)\right)=\operatorname{reg}\left(S / \operatorname{in}_{<}\left(J_{K_{m}}\right)\right)=$ $\operatorname{reg}\left(S / J_{K_{m}}\right)=1$, by [19, Theorem 2.1].

(2) Consider $\operatorname{in}_{<}\left(K_{m}, G\right)$ as an $m$-partite graph over the vertex set $W=\bigcup_{p=1}^{m} W_{p}$, where $W_{p}=\left\{x_{p 1}, \ldots, x_{p n}\right\}$, for all $p=1, \ldots, m$. It can be easily checked that for every $i, j$ with $1 \leqslant i<j \leqslant m$, the induced subgraph of $\operatorname{in}_{<}\left(K_{m}, G\right)$ on $W_{i, j}:=W_{i} \cup W_{j}$ is isomorphic to $\operatorname{in}_{<}(G)$. On the other hand, we have that $\operatorname{in}_{<}\left(K_{m}, G\right)=\bigcup_{1 \leqslant i<j \leqslant m}\left(\operatorname{in}_{<}\left(K_{m}, G\right)\right)_{W_{i j}}$. Thus, by Theorem 12 and Theorem 11, we obtain

$$
\operatorname{reg}\left(S / I\left(\operatorname{in}_{<}\left(K_{m}, G\right)\right)\right) \leqslant\left(\begin{array}{c}
m \\
2
\end{array}\right) \operatorname{reg}\left(S / I\left(\operatorname{in}_{<}(G)\right)\right) \leqslant\left(\begin{array}{c}
m \\
2
\end{array}\right) \operatorname{cochord}\left(\operatorname{in}_{<}(G)\right) .
$$

Now, similar to the proof of [19, Theorem 3.2], we show that $\operatorname{cochord}\left(\operatorname{in}_{<}(G)\right) \leqslant c(G)$. Let $H$ be a maximal clique of $G$. Then $\operatorname{in}_{<}(H)$ is an induced subgraph of $\operatorname{in}_{<}(G)$. By [19, Theorem 2.1], $I\left(\mathrm{in}_{<}(H)\right)$ has a linear resolution. Hence, by Fröberg's theorem, [4, Theorem 1], the complementary graph of $\operatorname{in}_{<}(H)$ is chordal. On the other hand, all maximal cliques of $G$, say $H_{1}, \ldots, H_{c(G)}$, cover all edges of $G$. So, clearly, in $\operatorname{in}_{<}\left(H_{1}\right), \ldots, \operatorname{in}_{<}\left(H_{c(G)}\right)$ cover all edges of $\operatorname{in}_{<}(G)$. Thus, by definition, we have cochord $\left(\operatorname{in}_{<}(G)\right) \leqslant c(G)$. Hence, $\operatorname{reg}\left(S / I\left(\operatorname{in}_{<}\left(K_{m}, G\right)\right)\right) \leqslant\left(\begin{array}{c}m \\ 2\end{array}\right) c(G)$.

Therefore, by the above two cases, we have

$$
\operatorname{reg}\left(I\left(\operatorname{in}_{<}\left(K_{m}, G\right)\right)\right)=\operatorname{reg}\left(S / I\left(\operatorname{in}_{<}\left(K_{m}, G\right)\right)\right)+1 \leqslant \min \left\{\left(\begin{array}{c}
m \\
2
\end{array}\right) c(G), e(G)\right\}+1,
$$

and hence the desired result follows, since $\operatorname{reg}\left(J_{K_{m}, G}\right) \leqslant \operatorname{reg}\left(\operatorname{in}_{<}\left(J_{K_{m}, G}\right)\right)$, by [5, Corollary 3.3.4].

Note that, by setting $m=2$, one could see that Theorem 13 yields the result of [19] on the regularity of the binomial edge ideal of a graph (see [19, Theorem 3.2]).

Corollary 14. Let $G$ be a closed graph on $[n]$ and $m, n \geqslant 2$. Then $\beta_{i, 2 i}\left(J_{K_{m}, G}\right)=0$, for all $i>\min \left\{\left(\begin{array}{c}m \\ 2\end{array}\right) c(G), e(G)\right\}+1$. In particular, we have $\beta_{i, 2 i}\left(J_{G}\right)=0$, for all $i>c(G)+1$.

Proof. Note that $\operatorname{reg}\left(J_{K_{m}, G}\right)=\max \left\{j-i: \beta_{i, j}\left(J_{K_{m}, G}\right) \neq 0\right\}$. So, if there exists some $i>\min \left\{\left(\begin{array}{c}m \\ 2\end{array}\right) c(G), e(G)\right\}+1$ with $\beta_{i, 2 i}\left(J_{K_{m}, G}\right) \neq 0$, then we have $\operatorname{reg}\left(J_{K_{m}, G}\right) \geqslant i$. Hence, $\operatorname{reg}\left(J_{K_{m}, G}\right)>\min \left\{\left(\begin{array}{c}m \\ 2\end{array}\right) c(G), e(G)\right\}+1$, which is a contradiction, by Theorem 13 . For the second part, it is enough to set $m=2$. 
The following corollary shows that the upper bound posed in Theorem 13 is sharp.

Corollary 15. Let $P_{n}$ be the path of length $n-1$ and $m \geqslant 2$ be an integer. Then we have $\operatorname{reg}\left(J_{K_{m}, P_{n}}\right)=n$. In particular, the regularity of $J_{K_{m}, P_{n}}$ does not depend on $m$.

Proof. Since $P_{n}$ is a closed graph, we can apply Theorem 13. So that we have $\operatorname{reg}\left(J_{K_{m}, P_{n}}\right) \leqslant \min \left\{\left(\begin{array}{c}m \\ 2\end{array}\right) c\left(P_{n}\right), e\left(P_{n}\right)\right\}+1=\min \left\{\left(\begin{array}{c}m \\ 2\end{array}\right)(n-1),(n-1)\right\}+1=n$. On the other hand, by Corollary 9 , part (b), we have $\operatorname{reg}\left(J_{P_{n}}\right) \leqslant \operatorname{reg}\left(J_{K_{m}, P_{n}}\right)$. But, one has $\operatorname{reg}\left(J_{P_{n}}\right)=n\left(\right.$ see $\left[19\right.$, Remark 3.3]). Therefore, $\operatorname{reg}\left(J_{K_{m}, P_{n}}\right)=n$, as desired.

Remark 16. The bound for the regularity in Theorem 13 might be strict. For instance, by using $\operatorname{CoCoA}$, one can see that $\operatorname{reg}\left(J_{K_{3}, K_{3}}\right)=3$, but Theorem 13 gives 4 as an upper bound.

The following corollary gives a lower bound for the regularity of the binomial edge ideal of a pair of graphs.

Corollary 17. Let $G_{1}$ and $G_{2}$ be two graphs on $[m]$ and $[n]$, respectively. If $p_{1}-1$ and $p_{2}-1$ are the lengths of the longest induced paths in $G_{1}$ and $G_{2}$, respectively, then we have $\operatorname{reg}\left(J_{G_{1}, G_{2}}\right) \geqslant \max \left\{p_{1}, p_{2}\right\}$.

Proof. It is enough to apply Proposition 8 and Corollary 15.

Corollary 18. Let $I$ be the ideal of adjacent 2-minors of an $m \times n$ generic matrix with $m \leqslant n$. Then we have $\operatorname{reg}(I) \geqslant n$.

We end this section by the following question about an upper bound for the regularity in a more general case, without the assumption of closedness:

Question. Let $G$ be a graph. Is it true that

$$
\operatorname{reg}\left(J_{K_{m}, G}\right) \leqslant \min \left\{\left(\begin{array}{c}
m \\
2
\end{array}\right) c(G), e(G)\right\}+1 ?
$$

In particular, by setting $m=2$, is it true that $\operatorname{reg}\left(J_{G}\right) \leqslant c(G)+1$ ?

Note that the latter bound is true if $G$ is a tree, as it was shown in [12] that for any graph $G$ on $n$ vertices, one has $\operatorname{reg}\left(J_{G}\right) \leqslant n$.

\section{Acknowledgements}

The authors thank Professor Jürgen Herzog for many useful discussions and also, the referee for his/her many useful comments. Further, the authors would like to thank to the Institute for Research in Fundamental Sciences (IPM) for financial support. The research of the second author was in part supported by a grant from IPM (No. 91050220). 


\section{References}

[1] M. Crupi and G. Rinaldo. Binomial edge ideals with quadratic Gröbner bases. Electron. J. Combin., 18 \#P211, 2011.

[2] V. Ene, J. Herzog and T. Hibi. Cohen-Macaulay binomial edge ideals. To appear in Nagoya Math. J. 204, 2011.

[3] V. Ene, J. Herzog, T. Hibi and A. A. Qureshi. The binomial edge ideal of a pair of graphs. Preprint, arXiv:1203.2775, 2012.

[4] R. Fröberg. On Stanley-Reisner rings. Topics in algebra., Banarch Center Publications, 26(2):57-70, 1990.

[5] J. Herzog and T. Hibi. Monomial ideals. Springer, 2010.

[6] J. Herzog and T. Hibi. Ideals generated by adjacent 2-minors. preprint, arXiv:1012.5789, 2011.

[7] J. Herzog, T. Hibi, F. Hreinsdotir, T. Kahle and J. Rauh. Binomial edge ideals and conditional independence statements. Adv. Appl. Math. 45:317-333, 2010.

[8] S. Hoşten and S. Sullivant. Ideals of adjacent minors. J. Algebra. 277:615-642, 2004.

[9] G. Kalai and R. Meshulam. Intersections of Leray complexes and regularity of monomial ideals. J. Combin. Theory Ser. A. 113 no. 7:1586-1592, 2006.

[10] M. Katzman. Characteristic-independence of Betti numbers of graph ideals. $J$. Combin. Theory Ser. A. 113:435-454, 2006.

[11] K. Kurano. The first syzygies of determinantal ideals. J. Algebra. 124:414-436, 1989.

[12] K. Matsuda and S. Murai. Regularity bounds for binomial edge ideals. Preprint, arXiv:1208.2415, 2012.

[13] M. Ohtani. Graphs and ideals generated by some 2-minors. Comm. Algebra. 39:905917, 2011.

[14] H. Ohsugi, J. Herzog and T. Hibi. Combinatorial pure subrings. Osaka J. Math. $37: 745-757,2000$.

[15] H. Ohsugi and T. Hibi. Toric ideals of finite graphs and adjacent 2-minors. Preprint, arXiv:1108.2850, 2012.

[16] I. Peeva. Graded syzygies. Springer, 2010.

[17] A. Rauf and G. Rinaldo. Construction of Cohen-Macaulay binomial edge ideals. to appear in Comm. Algebra.

[18] J. Rauh. Robustness and conditional independence ideals. Preprint, arXiv:1110.1338, 2011.

[19] S. Saeedi Madani and D. Kiani. Binomial edge ideals of graphs. Electron. J. Combin. 19(2):\#P44, 2012.

[20] T. Svanes. Coherent cohomology on Schubert subschemes of flag schemes and applications. Adv. Math. 14:369-453, 1974.

[21] R. H. Villarreal. Monomial Algebras. Marcel Dekker, 2001.

[22] R. Woodroofe. Matching, coverings, and Castelnuovo-Mumford regularity. Preprint, arXiv:1009.2756v2, 2011. 